\title{
Articles
}

\section{Age, body mass index and Type 2 diabetes- associations modified by ethnicity}

\author{
The DECODE-DECODA Study Group on behalf of the European Diabetes Epidemiology Group, \\ and the International Diabetes Epidemiology Group
}

c/o T. Nakagami, Steno Diabetes Centre, Gentofte, Denmark

\begin{abstract}
Aims/hypothesis. The aim of this study was to assess the effect of ethnicity on the association between age and body mass index as well as the prevalence of diabetes.

Methods. We selected population-based studies carried out after 1980 in the DECODE/A studies representing different ethnic groups: 11 European, 1 Maltese, 3 Indian, 2 Chinese and 3 Japanese surveys. The total numbers of subjects were 14,240 men and 15,129 women who were 30 to 89 years of age. Diabetes was diagnosed according to the 1999 World Health Organization criteria based on a standard 75 g OGTT. Sexspecific prevalence of diabetes by age and BMI was stratified by ethnic group, in particular the interaction of ethnicity on the associations between age/BMI and the prevalence of diabetes.
\end{abstract}

Results. The prevalence of diabetes was higher in studies from India and Malta compared to Japan, China, and the rest of Europe. The association between BMI and diabetes, adjusted for age, showed noticeable differences between the ethnic groups with an increase in prevalence starting at a BMI between 15 and $20 \mathrm{~kg} / \mathrm{m}^{2}$ in the Maltese and Indian populations compared to $25 \mathrm{~kg} / \mathrm{m}^{2}$ in Europeans.

Conclusion/interpretation. The effect of BMI on the age-adjusted prevalence of Type 2 diabetes was modified by ethnicity with considerably lower thresholds in Indian and Maltese subjects compared to those from the rest of Europe. This difference should be reflected in national and international recommendations regarding “optimal” BMI. [Diabetologia (2003) 46:1063-1070]

Keywords Diabetes, prevalence, ethnic, BMI, age, DECODE, DECODA
Type 2 diabetes mellitus is among the fastest growing diseases in the world due to a combination of unfavourable changes in modifiable risk factors and a high

Received: 30 December 2002 / Revised: 25 April 2003

Published online: 25 June 2003

(C) Springer-Verlag 2003

Corresponding author: The DECODE-DECODA Study Group, c/o T. Nakagami, Steno Diabetes Centre, Niels Steensense Vej 2, 2820 Gentofte, Denmark

E-mail: nakagami@dmc.twmu.ac.jp

Abbreviations: DECODE, Diabetes Epidemiology Collaborative analysis of Diagnostic criteria in Europe; DECODA, Diabetes Epidemiology Collaborative analysis of Diagnostic criteria in Asia; WHO, World Health Organization.

Members of the DECODE-DECODA study group are listed at the end of this paper genetic susceptibility in many populations [1]. The rapid increase of diabetes worldwide is primarily a consequence of aging in most populations and the increase of obesity and physical inactivity [2].

Cross-sectional $[3,4,5]$ and prospective $[6,7,8]$ studies have shown an association between age and body mass index and the prevalence and incidence of Type 2 diabetes. These associations have been consistently reported from different ethnic groups.

The prevalence of Type 2 diabetes varies considerably between countries, regions and ethnic groups [2]. Although the variation in prevalence has been associated with regional variations in the distribution of established behavioral and environmental risk factors [3, $4,5]$, it has not been fully explained by these factors.

The aim of this study was to assess the effect of ethnicity on the association between age, BMI, and 
Table 1. Characteristics of survey analysed for this study

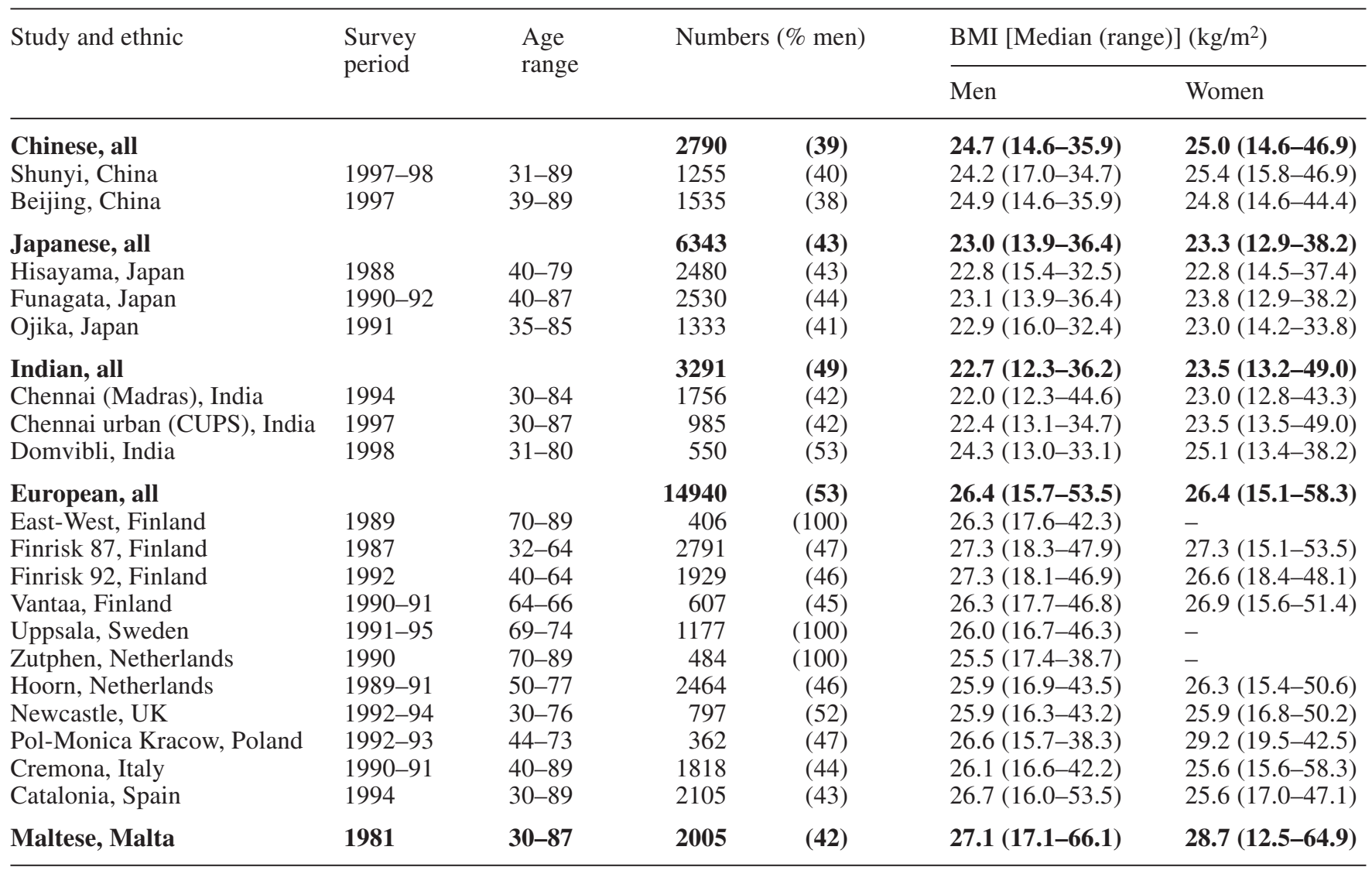

the prevalence of Type 2 diabetes based on the analysis of data from two collaborative studies, "Diabetes Epidemiology: Collaborative analysis of Diagnostic Criteria in Europe (DECODE)" and "Diabetes Epidemiology: Collaborative Analysis of Diagnostic Criteria in Asia (DECODA)" [9, 10].

\section{Subjects and methods}

Participants and study design. We invited centres in Europe and Asia, who had done surveys on the prevalence of Type 2 diabetes, using a standard 75-g OGTT with fasting and 2-h glucose measurements, to participate in the DECODE and DECODA studies. The study design and data collection have been described in detail in previous publications $[9,10]$. Each centre provided original data including age, sex, date of examination, height, weight, status of known diabetes, glucose concentrations (fasting and 2-h), blood specimen used (venous whole blood, venous plasma, serum or capillary whole blood) and the method of the glucose assay used. BMI was calculated as weight $(\mathrm{kg}) / \mathrm{height}^{2}(\mathrm{~m})$. Data were sent to the Diabetes and Genetic Epidemiology Unit at the National Public Health Institute in Helsinki, Finland.

The inclusion criteria for this paper were: population-based studies carried out after 1980 using a standard 2-h 75-g OGTT in the morning after an overnight fast of at least $10 \mathrm{~h}$ according to the World Health Organization (WHO) recommendations [11]. Twelve surveys from Europe [12, 13, 14, 15, 16, 17, 18] including a survey from Malta, three surveys from India [19,
20, 21], two surveys from China and three surveys from Japan $[22,23,24]$ fulfilled these criteria and were included in the study. A total of 29,369 subjects (14,240 men and 15,129 women) who were 30 to 89 years of age were included in the analysis (Table 1). Data for Malta were analysed separately from the other European countries for two reasons: firstly, there was a 10-year difference in the survey period between Malta and the other European surveys and secondly, the ageadjusted prevalence of the Maltese population was very high compared to the other European populations [25].

Subjects who had previously been diagnosed with Type 2 diabetes or those who were newly-diagnosed with a fasting plasma glucose over $7.0 \mathrm{mmol} / \mathrm{l}$ and/or a 2-h plasma glucose over $11.1 \mathrm{mmol} / \mathrm{l}$ after a 75-g OGTT according to the WHO criteria [26], were considered as having diabetes. For surveys using serum, venous whole blood or capillary whole blood, the corresponding diagnostic cut-points were: $7.0,6.1$ and $6.1 \mathrm{mmol} / \mathrm{l}$ (fasting) and 11.1, 10.0 and $11.1 \mathrm{mmol} / \mathrm{l}$ (2-h post load).

Statistical analysis. All analyses were carried out and are reported by sex. The sex-specific mean levels of BMI stratified by age were compared between ethnic groups by ANOVA in 10 -year age group strata. The logistic regression model was adopted to estimate the prevalence of diabetes (log odds (diabetes) $=$ intercept $+\alpha$ age $+\gamma$ ethnicity) with age as a continuous variable and ethnicity classified as European, Maltese, Indian, Chinese and Japanese. The prevalence of diabetes adjusted for age and BMI was estimated using a spline model with age, BMI and ethnicity included as explanatory variables and where age of 45, 55, 60 and 70 years and BMI of 22, 24, 26 and $28 \mathrm{~kg} / \mathrm{m}^{2}$ were adopted as knots (for details see equation in Appendix 1). The interaction effect of ethnicity and age/BMI 

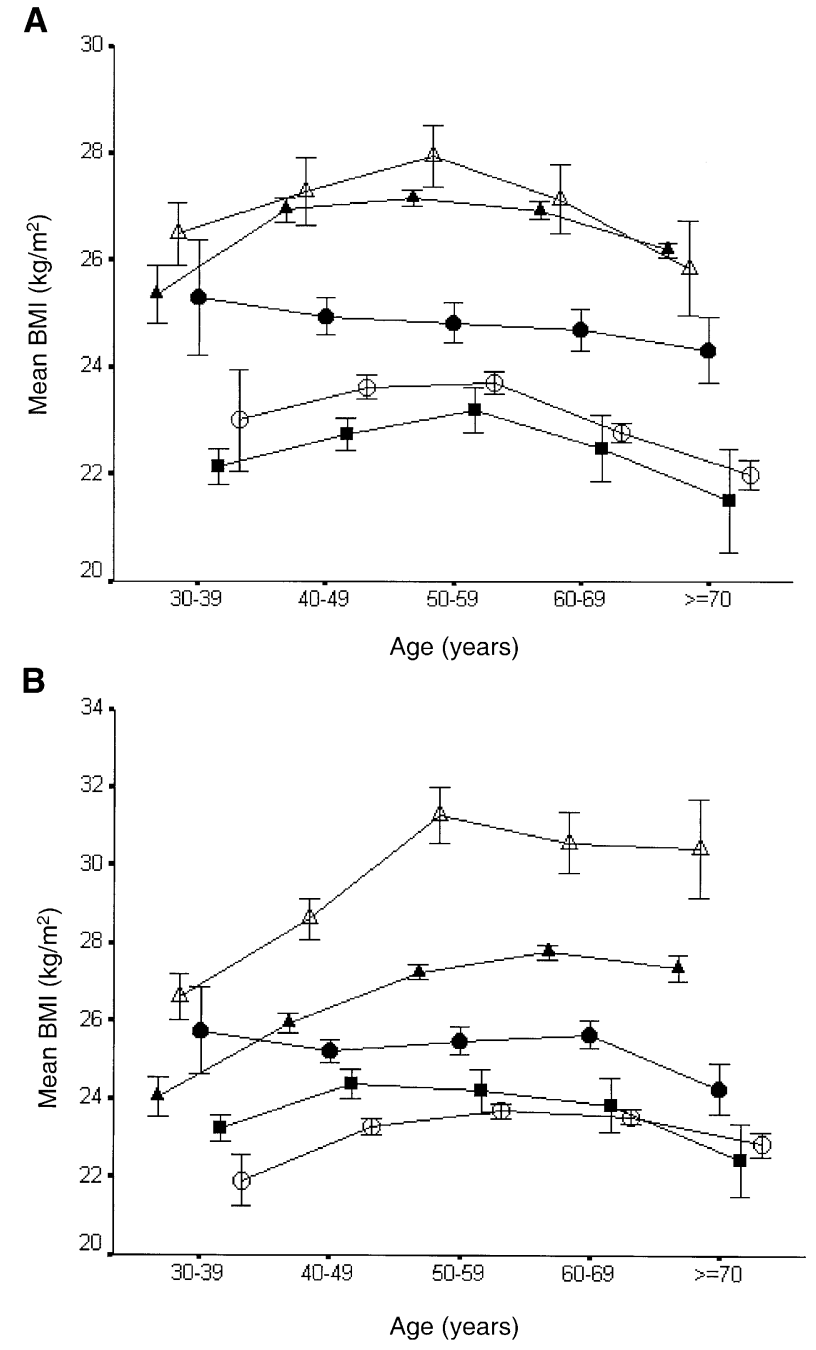

Fig. 1A, B. Mean body mass index (BMI) with 95\% confidence intervals (CI) by age and ethnic group, (A) for men and (B) for women. European (closed triangles), Maltese (open triangles), Indian (closed squares), Chinese (closed circles) and Japanese (open circles)

was done by using the chi-squared log likelihood ratio test with nested models. Similar statistical analyses were done to test the interaction effect of sex on the association between age, BMI and prevalence of diabetes within the same ethnic group. All statistical analysis was done by using SPSS for Windows version 11.0. A $p$ value of less than 0.05 was considered to be statistically significant. All participants gave their informed consent to participate in the study according to the declaration of Helsinki and other ethical standards relevant to the time when the data was collected.

\section{Results}

Table 1 shows the basic characteristics for each survey. The age ranged from 30 to 89 years, but with pronounced difference between the surveys. The lowest BMI was found in Chennai (Madras), India (median $22.0 \mathrm{~kg} / \mathrm{m}^{2}$ in men and $23.0 \mathrm{~kg} / \mathrm{m}^{2}$ in women) and the highest BMI in Finrisk 92, Finland $\left(27.3 \mathrm{~kg} / \mathrm{m}^{2}\right.$ in
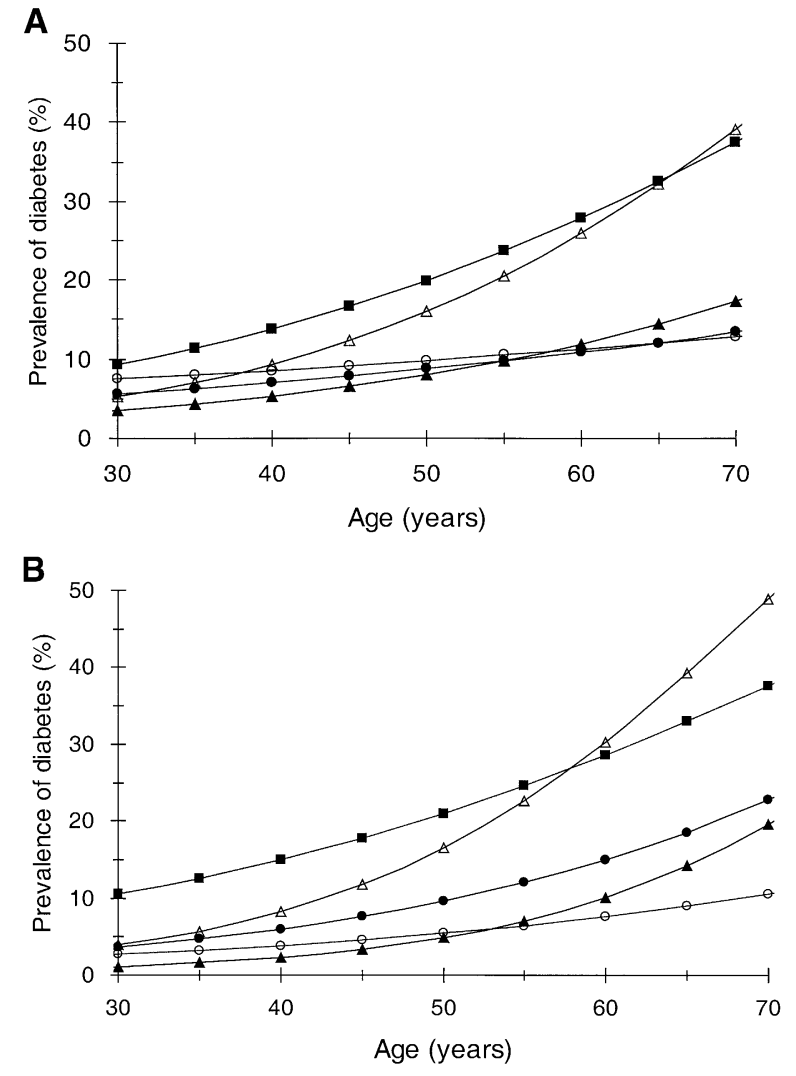

Fig. 2A, B. Age-specific prevalence of diabetes by age and ethnic group, (A) for men and (B) for women. European (closed triangles), Maltese (open triangles), Indian (closed squares), Chinese (closed circles) and Japanese (open circles)

men and women) and Malta $\left(27.1 \mathrm{~kg} / \mathrm{m}^{2}\right.$ in men and $28.7 \mathrm{~kg} / \mathrm{m}^{2}$ in women). In both sexes age-specific BMI was highest in Malta and Europe with the lowest levels found in Japan and India (Fig. 1). Except for China, BMI increased by age until age 50 to 59 years in men and 60 to 69 years in women; thereafter it stabilised or decreased.

Prevalence of diabetes by age. In all ethnic groups the prevalence of diabetes increased with age, but the association between age and prevalence of diabetes was higher in the Indian and Maltese populations compared to all other ethnic groups in both men and women (Fig. 2). The association between age and prevalence of diabetes differed significantly between the ethnic groups, with the increase by age being higher in the Maltese $(p<0.05)$ and lower for the Chinese $(p<0.05)$ and Japanese $(p<0.005)$ cohorts compared to the European cohorts. By the age of 60 years the estimated prevalence of diabetes in men and women were $27.9 / 28.6 \%$ in Indian, $25.9 / 29.2 \%$ in Maltese, $11.9 / 10.1 \%$ in European, 11.2/7.6\% in Japanese, and 10.9/15.0\% in Chinese subjects.

Interaction between ethnicity, age, BMI and prevalence of Type 2 diabetes. Adjusted for age, the associ- 

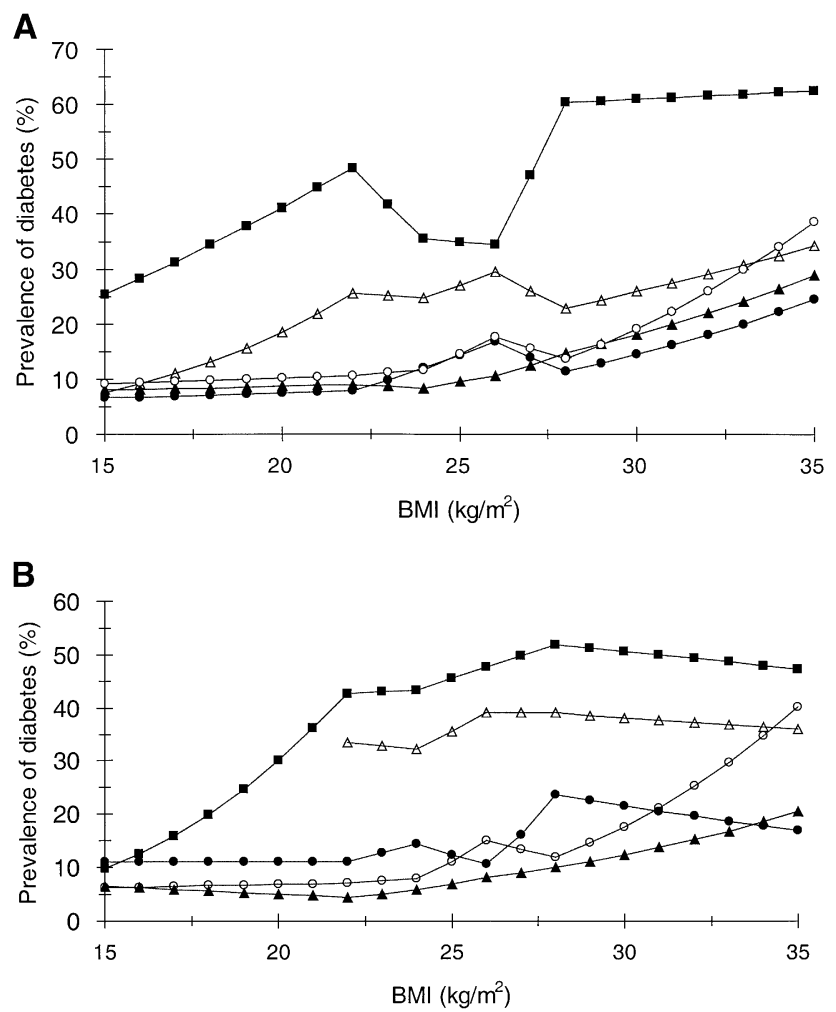

Fig. 3A, B. BMI and prevalence of DM, (A) for 60-year-old men and (B) for 60-year-old women. European (closed triangles), Maltese (open triangles), Indian (closed squares), Chinese (closed circles) and Japanese (open circles)

ation between BMI and prevalence of diabetes differed significantly between the ethnic groups with the highest prevalence in Indian subjects followed by Maltese, Japanese, European and Chinese men while in women it was highest in Indian, followed by Maltese, Chinese, Japanese and European women (Fig. 3): In men using Europe as the reference age adjusted effect of BMI on the prevalence of diabetes differed significantly in Malta $(p<0.01)$ and India $(p<0.05)$, while in women it differed in Malta $(p<0.001)$, India $(p<0.001)$ and China $(p<0.005)$. In the Indian cohort the age-adjusted prevalence of diabetes started to increase in the BMI range of 15 to $20 \mathrm{~kg} / \mathrm{m}^{2}$ for both men and women. In the Maltese cohort the prevalence increased with BMI in men but not in women, whereas in all the three other groups the prevalence increased by BMI in both sexes. The sex difference in the effect of BMI on the age-adjusted prevalence of diabetes was significant only in the Indian cohort $(p<0.01)$.

Figure 4 shows the effect of age on the prevalence of diabetes in men and women adjusted for BMI (BMI set at $25 \mathrm{~kg} / \mathrm{m}^{2}$ ). In men the European and Chinese prevalence curves started to increase by the age of 45 while for all the other three groups the prevalence started to increase by the age of 30 years. In European women the prevalence of diabetes started to increase
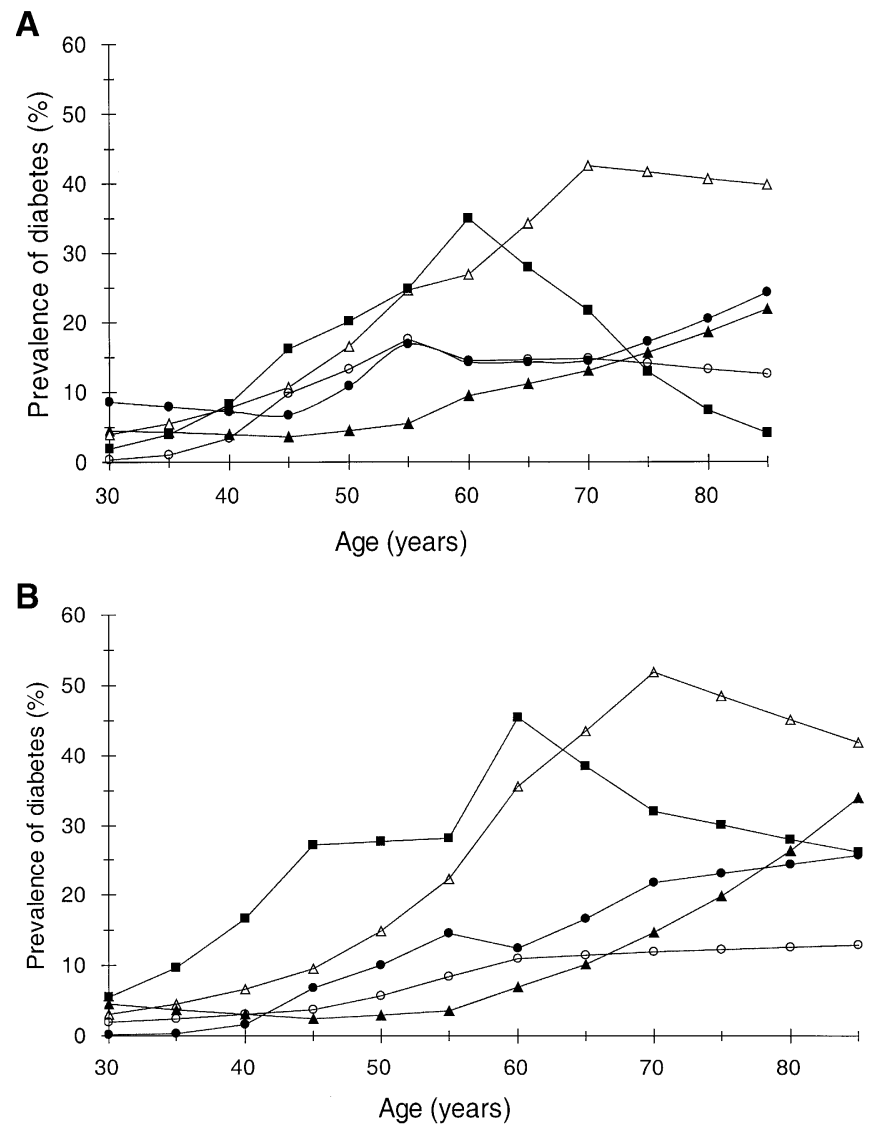

Fig. 4A, B. Age and prevalence of DM, (A) for men with BMI $25 \mathrm{~kg} / \mathrm{m}^{2}$ and (B) for women with BMI $25 \mathrm{~kg} / \mathrm{m}^{2}$. European (closed triangles), Maltese (open triangles), Indian (closed squares), Chinese (closed circles) and Japanese (open circles)

by age 45 years, while in all other ethnic groups the increase started between 30 and 40 years of age. The peak prevalence was found by the age of 55 to 60 years in Japanese, 60 years in Indian and 70 years in Maltese groups independent of sex, while the prevalence continued to increase throughout all ages in the Chinese and European groups. The BMI-standardized age effect differed between the ethnic groups. In men, using European as the reference the BMI adjusted effect of age on the prevalence of diabetes was significantly different in the Indian, Chinese and Japanese men ( $p<0.001, p<0.05, p<0.001$ respectively), and in women the Maltese, Indian, Chinese and Japanese women all differed significantly from the European women ( $p<0.001$ for all comparison). The BMIadjusted effect of age on the prevalence of diabetes differed between men and women in the European $(p<0.001)$, Chinese $(p<0.05)$ and Japanese $(p<0.05)$ cohorts.

\section{Discussion}

This study confirms the large ethnic, regional and sexdependent variation in the age-adjusted prevalence of 
Type 2 diabetes as reviewed by the WHO [2]. This study also shows that this variation cannot be explained by differences in BMI between the populations. We found that ethnicity modified the effect of BMI on the age-adjusted prevalence of Type 2 diabetes. The association between age and the prevalence of diabetes was also modified by ethnicity. Together these observations indicate that ethnicity not only affects the over all susceptibility to diabetes, but also modifies the effect of established risk factors for Type 2 diabetes. Ethnicity is associated with a long list of factors including genetic constitution, living conditions, life style factors and anthropometry. Thus it is not simply the difference in BMI distributions that explain the worldwide variation in the agestandardized prevalence of Type 2 diabetes.

The relative contribution of reduced insulin secretion and reduced insulin sensitivity as contributors to the development of diabetes differs between various ethnic groups. Asian Indians have been shown to be more insulin-resistant than most other ethnic groups [27]. Preferential deposition and distribution of fat in the abdominal region is associated with reduced insulin-mediated glucose disposal, independent of an overall degree of obesity [28, 29, 30]. Anthropometric studies have shown that the distribution of fat differs between ethnic groups, and thus identical distributions of BMI in two populations could still reflect large differences with respect to the accumulation of intraabdominal fat, and Asian populations have been shown to be more prone to abdominal obesity and low muscle mass [27, 31, 32, 33]. Thus excessive insulin resistance related to intra-abdominal fat deposition and low muscle mass could explain the very high prevalence of diabetes in the Indian populations despite the absence of significant obesity as expressed by BMI.

Indians had a very high prevalence of diabetes adjusted for age, and the risk started to increase at very low levels of BMI. The explanation should probably partly be found through the same explanations as those given for Chinese and Japanese subjects, but in addition to this there could also be an association with the very low birth weight often seen in Indian subjects [34]. It has been suggested that low birth weight as an indicator of intrauterine malnutrition could be an important contributor to the otherwise peculiar prevalence pattern found in Indian subjects [35]. A recent study carried out in India has confirmed the importance of intrauterine and early childhood development for the risk of Type 2 diabetes [34].

In contrast, insulin deficiency rather than insulin resistance has been reported to play a prominent role in the progression of diabetes in Japanese subjects [36]. Thus the insulin secretory inferiority could partly explain the higher prevalence of diabetes in non-obese Japanese and Chinese populations compared to European populations.
Our finding that the association between BMI and diabetes is modified by ethnicity supports the need for a redefinition of obesity based on geographical region and ethnicity as suggested by the joint enterprise of the Regional Office for the Western Pacific of the WHO, the International Association for the Study of Obesity and the International Obesity Task Force in 2000 [37]. Although BMI is a simple and non-invasive method for assessing obesity and excessive fat stores within a single population, it is less reliable when it comes to comparing body composition between populations $[38,39]$. Our findings indicate that $\mathrm{BMI}$ is an unreliable measure for studying the association between obesity and insulin resistance or Type 2 diabetes across ethnic groups. Further studies should focus on the potential ethnic differences in the regional fat distribution, and simple and reliable instruments are needed to evaluate the metabolic consequences of visceral fat obesity between geographical regions and ethnic groups. The simple measurement of waist circumference could be a suitable measure.

We found that the overall effect of age on the prevalence of diabetes differed considerably between the ethnic groups even in subjects with the same BMI. Moreover we found that the prevalence started to increase at a higher age in the European cohort than in any other group studied. These observations must, however, be interpreted with some caution as differences in mortality between ethnic groups will contribute to differences in the prevalence of diabetes. The delayed clinical diagnosis in the Chinese and Japanese populations combined with the excess mortality associated with Type 2 diabetes [40] could explain the lower prevalence of diabetes in the old age groups in some Asian populations.

The increasing prevalence of diabetes worldwide has been labelled an "epidemic of diabetes" and at present the focus is on how this "epidemic" can be prevented. Three different studies have shown that dietary modification and increased physical activity can reduce the 6-year risk of developing diabetes by 40 to $60 \%$ in high-risk subjects with IGT [41, 42, 43]. These recent trials have provided guidelines as to who should be targeted in the preventive process. Our data showing that ethnicity has a major effect on the deleterious consequences of obesity with aging suggests that target groups for intervention strategies (based on age and BMI) may have to be defined for each ethnic group separately, and future studies should aim at defining these high risk groups.

Acknowledgements. Japan Diabetes Foundation and Japan Arteriosclerosis Prevention Fund (Japanese Ministry of Health, Welfare and Labour) supported a fellowship for T. Nakagami. The DECODE/A study has been carried out by the support of grants from Novartis Pharma AG, Basel Switzerland and the Finnish Academy (grants 46558, 76502 and 77618). The DECODE Study was funded by Novo Nordisk, Bagsværd, Denmark. 


\section{Appendix 1}

The prevalence of diabetes adjusted for age and BMI was estimated using the model with spline form in which age, BMI and ethnicity were included as explanatory variables as follows:

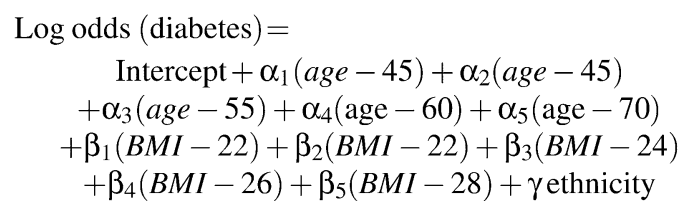

Age-45: $\quad$ subtract 45 from age (years)

Age-45: When age was $<45$ years old, replace "0". Otherwise, subtract 45 from age (years)

Age-55: When age was $<55$ years old, replace " 0 ". Otherwise, subtract 55 from age (years)

Age-60: When age was $<60$ years old, replace "0". Otherwise, subtract 60 from age (years)

Age-70: When age was $<70$ years old, replace " 0 ". Otherwise, subtract 70 from age (years)

BMI-22: $\quad$ subtract 22 from BMI $\left(\mathrm{kg} / \mathrm{m}^{2}\right)$

BMI-22: When BMI was $<22 \mathrm{~kg} / \mathrm{m}^{2}$, replace " 0 ". Otherwise, subtract 22 from BMI $\left(\mathrm{kg} / \mathrm{m}^{2}\right)$

BMI-24: When BMI was $<24 \mathrm{~kg} / \mathrm{m}^{2}$, replace " 0 ". Otherwise, subtract 24 from BMI $\left(\mathrm{kg} / \mathrm{m}^{2}\right)$

BMI-26: When BMI was $<26 \mathrm{~kg} / \mathrm{m}^{2}$, replace " 0 ". Otherwise, subtract 26 from BMI $\left(\mathrm{kg} / \mathrm{m}^{2}\right)$

BMI-28: When BMI was $<28 \mathrm{~kg} / \mathrm{m}^{2}$, replace "0". Otherwise, subtract 28 from BMI $\left(\mathrm{kg} / \mathrm{m}^{2}\right)$

In this model, age at 45, 55, 60 and 70 years and BMI at 22, 24, 26 and $28 \mathrm{~kg} / \mathrm{m}^{2}$ were adopted as knots where the log odds for having diabetes would be changed. With Europe as the reference group the ethnic interaction effect of age/BMI on the prevalence of diabetes was tested by the log likelihood ratio between the two models with and without the variable of "age/BMI*ethnicity".

\section{Appendix 2}

The DECODE Study (Diabetes Epidemiology: Collaborative Analysis of Diagnostic Criteria in Europe) was undertaken on the initiative of the European Diabetes Epidemiology Group. The DECODA Study (Diabetes Epidemiology: Collaborative Analysis of Diagnostic Criteria in Asia) was undertaken on the initiative of the International Diabetes Epidemiology Group.

Study centres included in this analysis are

\section{Finland}

- East-West Finland Study: A. Nissinen, J. Tuomilehto, Department of Epidemiology and Health Promotion, National Public Health Institute, Helsinki, Finland.

- Finrisk Study: J. Tuomilehto, J. Lindström, P. Jousilahti, Department of Epidemiology and Health Promotion, National Public Health Institute, Helsinki, Finland.

- Vantaa Study: R. Tilvis, J. Tuomilehto, Department of Epidemiology and Health Promotion, National Public Health Institute, Helsinki, Finland.
Italy

- Cremona Study: G. Gallus, M. Paora Garancini, Epidemiology Unit, San Raffaele Institute, Milan.

Malta

- Malta Study: A. Schranz, D. Clinic, St Luke's Hospital, M'Gania

Poland

- POL-MONICA Study (Krakow): A. Pajak, Department of Clinical Epidemiology and Population Studies, Institute of Public Health, Collegium Medicum, Jagiellonian University, Krakow.

Spain

- Catalonia Study: C. Castell, G. Lloveras, R. Tresserras. Department of Health and Social Security. The Autonomous Government of Catalonia.

Sweden

- Uppsala Study: H. Lithell, B. Zethelius, Department of Geriatrics, University of Uppsala, Uppsala.

\section{Netherlands}

- Hoorn Study: R. J. Heine, J. M. Dekker on behalf of the Hoorn Study Research Group, Institute of Research in Extramural Medicine, Vrije Universiteit.

- Zutphen Elderly Study: E. J. M. Feskens, D. Kromhout, National Institute of Public Health and Environment, Bitlhoven.

$U K$

- Newcastle Heart Study: N. Unwin, N. Ahmad, K. George M. M. Alberti, Department of Medicine and Public Health, University of Newcastle, Newcastle.

People's Republic of China

- Shunyi Study: Z. Yang, Institute of Geriatrics and Beijing Hospital, Ministry of Health, Beijing. Bingzhi Wang, Shunyi County People's Hospital, Shunyi, Henan.

- Beijing Study: Z. Yang, Z. Tong, Institute of Geriatrics and Beijing Hospital, Ministry of Health, Beijing. Qinying Fan, China-Japan Friendship Hospital, Ministry of Health, Beijing.

India

- Madras (Chennai) Study: A. Ramachandran, Diabetes Research Centre, Chennai.

- Chennai Urban Population Study (CUPS): V. Mohan, C. S. Shanthi Rani, R. Deepa, Madras Diabetes Research Foundation, Chennai.

- Dombivli Study: S. Ramnathan Iyer, Ambika Clinic and Research Centre, Dombivli (East), District Thane, Maharashtra.

Japan

- Ojika Survey: M. Nagai, S. Shibazaki, Department of Public Health, Saitama Medical School, Saitama.

- Hisayama Study: M. Fujishima, Y. Kiyohara, Second Department of Internal Medicine, Faculty of Medicine, Kyushu University, Fukuoka.

- Funagata Diabetes Studies: M. Tominaga, Department of Laboratory Medicine, Yamagata University, Yamagata, Japan 


\section{Data analysis}

T. Nakagami, K. Borch-Johnsen and Bendix Carstensen, Steno Diabetes Centre, Gentofte, Denmark.

\section{Writing Committee}

T. Nakagami ${ }^{1}$, Q. Qiao ${ }^{2}$, B. Carstensen ${ }^{1}$, C. Nøhr-Hansen ${ }^{1}$, G. $\mathrm{Hu}^{2}$, J. Tuomilehto ${ }^{2}$, B. Balkau ${ }^{3}$ and K. Borch-Johnsen ${ }^{1}$

${ }^{1}$ Steno Diabetes Centre, Gentofte, Denmark; ${ }^{2}$ Department of Epidemiology and Health Promotion, National Public Health Institute, Helsinki, Finland; ${ }^{3}$ INSERM U258, Villejuif, France.

\section{References}

1. Neel JV (1962) Diabetes mellitus "thrifty" genotype rendered detrimental by "process"? Am J Hum Genet 14:353362

2. King H, Rewers M, WHO AD HOC Diabetes Reporting Group (1993) Global estimates for prevalence of diabetes mellitus and impaired glucose tolerance in adults. Diabetes Care 16:157-177

3. Midthjell K, Knuger Ø, Holmen J et al. (1999) Rapid change in the prevalence of obesity and known diabetes in an adult Norwegian population. The third-Trøndelag Health Surveys: 1984-1986 and 1995-1997. Diabetes Care 22:1813-1820

4. Fujimoto WY, Leonetti DL, Kinyoun JL et al. (1987) Prevalence of diabetes mellitus and impaired glucose tolerance among second-generation Japanese-American men. Diabetes 36:721-729

5. Omar MA, Seedat MA, Dyer RB, Motala AA, Knight LT, Becker PJ (1994) South African Indians show a high prevalence of NIDDM and bimodality in plasma glucose distribution patterns. Diabetes Care 17:70-73

6. Haffner SM, Stern MP, Mitchell BD, Hazuda HP, Patterson JK (1990) Incidence of type II diabetes in Mexican Americans predicted by fasting insulin and glucose levels, obesity, and body-fat distribution. Diabetes 39:283-288

7. Everhart JE, Pettitt DJ, Bennett PH, Knowler WC (1992) Duration of obesity increases the incidence of NIDDM. Diabetes 41:235-240

8. Colditz GA, Willett WC, Rotnitzky A, Manson JE (1995) Weight gain as a risk factor for clinical diabetes mellitus in women. Ann Intern Med 122:481-486

9. DECODE Study Group on behalf of the European Diabetes Epidemiology Study Group (1998) Will new diagnostic criteria for diabetes mellitus change phenotype of patients with diabetes? Reanalysis of European epidemiological data. BMJ 317:371-375

10. DECODA Study Group on behalf of the International Diabetes Epidemiology Group (2000) Comparison of the fasting and the 2-hour glucose criteria for diabetes in different Asian cohorts. Diabetologia 43:1470-1475

11. World Health Organization Expert Committee (1980) Diabetes Mellitus: Second report. WHO Technical Report Series, No. 646, WHO, Geneva

12. Stegmayr B, Asplund K (1995) Diabetes as a risk factor for stroke. A population perspective. Diabetologia 38:10611068

13. Skarfors ET, Selinus KI, Lithell HO (1991) Risk factors for developing non-insulin dependent diabetes: a 10 year follow up of men in Uppsala. BMJ 303:755-760
14. Pyorala K, Savolainen E, Lehtovirta E, Punsar S, Siltanen P (1979) Glucose tolerance and coronary heart disease: Helsinki policemen study. Glucose tolerance and coronary heart disease: Helsinki policemen study. J Chronic Dis. 32:729-745

15. Garancini MP, Calori G, Ruotolo G et al. (1995) Prevalence of NIDDM and impaired glucose tolerance in Italy: an OGTT-based population study. Diabetologia 38:306-313

16. Vartiainen E, Puska P, Jousilahti P, Korhonen HJ, Tuomilehto J, Nissinen A (1994) Twenty-year trends in coronary risk factors in north Karelia and in other areas of Finland. Int J Epidemiol 23:495-504

17. Stengard JH, Pekkanen J, Tuomilehto J et al. (1993) Changes in glucose tolerance among elderly Finnish men during a five-year follow-up: the Finnish cohorts of the Seven Countries Study. Diabet Metab19:121-129

18. Schranz A, Tuomilehto J, Marti B, Jarrett RJ, Grabauskas V, Vassallo A (1991) Low physical activity and worsening of glucose tolerance: results from a 2-year follow-up of a population sample in Malta. Diabetes Res Clin Pract 11:127-136

19. Mohan V, Shanthi Rani CS, Deepa R, Premalatha G, Sastry NG, Saroja R (2001) Intra urban differences in the prevalence of metabolic syndrome in southern India-The Chennai Urban Population Study (CUPS). Diabet Med 18:280-287

20. Ramachandran A, Snehalatha C, Latha E, Vijay V, Viswanathan M (1997) Rising prevalence of NIDDM in an urban population in India. Diabetologia 40:232-237

21. Iyer SR, Iyer RR, Upasani SV, Baitule MN (2001) Diabetes mellitus in Dombivli-an urban population study. J Assoc Physicians India 49:713-716

22. Ohmura T, Ueda K, Kiyohara Y et al. (1993) Prevalence of Type 2 (non-insulin-dependent) diabetes mellitus and impaired glucose tolerance in the Japanese general population: the Hisayama study. Diabetologia 36:1198-1203

23. Nagai M, Sakata K, Yanagawa H, Sueta H, Tanaka T, Shirahama S (1992) The prevalence of diabetes mellitus and impaired glucose tolerance studies by 75 -gram oral glucose tolerance test in a rural island population. Japanese Journal of Public Health 39:907-912

24. Sekikawa A, Tominaga M, Takahashi K et al. (1993) Prevalence of diabetes and impaired glucose tolerance in Funagata area, Japan. Diabetes Care 16:570-574

25. DECODE Study Group on behalf of the European Diabetes Epidemiology Study Group (2003). Age- and sex- specific prevalence of diabetes and impaired glucose regulation in 13 European cohorts. Diabetes Care 26:61-69

26. World Health Organization (1999) Definition, Diagnosis and Classification of Diabetes Mellitus and its Complications. Report of a WHO Consultation, Part1: Diagnosis and Classification of Diabetes Mellitus. WHO, Geneva (WHO/NCD/NCS/99.2)

27. Abate N, Chandalia M (2001) Ethnicity and type 2 diabetes: focus on Asian Indians. J Diabetes Complications 15:320-327

28. Despres JP, Nadeau A, Tremblay A et al. (1989) Role of deep abdominal fat in the association between regional adipose tissue distribution and glucose tolerance in obese women. Diabetes 38:304-309

29. Abate N, Garg A, Pershock RM, Stray-Gundersen J, Grundy SM (1995) Relationships of generalized and regional adiposity to insulin sensitivity in men. J Clin Invest 96:88-98

30. Goodpaster BH, Thaete FL, Simoneau JA, Kelley DE (1997) Subcutaneous abdominal fat and thigh muscle composition predict insulin sensitivity independently of visceral fat. Diabetes 46:1579-1585 
31. Mckeigue PM, Pierpoint T, Ferrie JE, Marmont MG (1992) Relationship of glucose intolerance and hyperinsulinemia to body fat pattern in South Asians and Europeans. Diabetologia 35:785-791

32. Karter AJ, Mayer-Davis EJ, Selby JV et al. (1996) Insulin sensitivity and abdominal obesity in African-American, Hispanic, and non-Hispanic white men and women. The Insulin Resistance and Atherosclerosis Study. Diabetes 45:1547-1555

33. Wang J, Thornton JC, Russel M, Burastero S, Heymsfield S (1994) Asians have lower body mass index (BMI) but higher percent body fat than do whites: comparisons of anthropometric measurements. Am J Clin Nutr 60:23-28

34. Yajnik CS, Fall CHD, Vaidya U et al. (1995) Fetal growth and glucose and insulin metabolism in four-year-old Indian children. Diabet Med 12:330-336

35. Barker DJP, Hales CN, Fall CHD, Osmond C, Phipps K, Clark PMS (1993) Type2 (non-insulin dependent) diabetes mellitus, hypertension and hyperlipidemia (syndrome $\mathrm{X}$ ): relation to reduced fetal growth. Diabetologia 36:62-67

36. Matsumoto K, Miyake S, Yano M et al. (1997) Glucose tolerance, insulin secretion, and insulin sensitivity in nonobese and obese Japanese subjects. Diabetes Care 20:1562-1568

37. Regional Office for the Western Pacific of the World Health Organization, International Association for the
Study of Obesity and the International Obesity Task Force (2000) The Asia-Pacific perspective: Redefining obesity and its treatment. (http://www.obesityasiapacific.com/) Health Communications Australia:22-29

38. Norgan NG (1994) Population differences in body composition in relation to body mass index. Eur J Clin Nutr 48 (Suppl):10-27

39. Deurenberg P, Deurenberg Yap M, Wang J, Lin FP, Schmidt G (1999) The impact of body build on the relationship between body mass index and percent body fat. Int J Obes Relat Metab Disord 23:537-542

40. Yokoyama H, Okudaira M, Otani T et al. (1998) High incidence of diabetic nephropathy in early-onset Japanese NIDDM patients. Diabetes Care 21:1080-1085

41. Pan XR, Li GW, Hu YH et al. (1997) Effects of diet and exercise in preventing NIDDM in people with impaired glucose tolerance. The Da Qing IGT and diabetes study. Diabetes Care 20:537-544

42. Eriksson KF, Lindgärde F (1991) Prevention of Type 2 (non-insulin-dependent) diabetes mellitus by diet and physical exercise. The 6-year Malmö feasibility study. Diabetologia 34:891-898

43. Tuomilehto J, Lindström J, Eriksson JG et al. (2001) Prevention of Type 2 diabetes by changes in lifestyle among subjects with impaired glucose tolerance. N Eng J Med 344:1343-1350 